\title{
A Case-control Study on Personal and Academic Determinants of Dropout among Health Profession Students
}

\author{
Thamir Aldahmashi ${ }^{1,2}$, Thekra Algholaiqa ${ }^{1}$, Ziyad Alrajhi ${ }^{1,3}$, Thamer Althunayan ${ }^{1}, \operatorname{Irfan}$ Anjum ${ }^{1} \&$ Bader \\ Almuqbil ${ }^{1}$ \\ ${ }^{1}$ King Saud bin Abdulaziz University for Health Sciences, Riyadh, Saudi Arabia \\ ${ }^{2}$ Department of ENT, King Abdullah bin Abdulaziz University Hospital, Riyadh, Saudi Arabia \\ ${ }^{3}$ Department of Paediatric Hematology-Oncology, King Faisal Specialist Hospital and Research Centre, Riyadh, \\ Saudi Arabia \\ Correspondence: Thamir Aldahmashi, Riyadh, P.O. Box: 14235, Saudi Arabia. Tel: 966-565-539-958. E-mail: \\ dr.thamir2012@gmail.com. https://orcid.org/0000-0001-6170-1458
}

Received: January 24, 2021

Accepted: March 17, $2021 \quad$ Online Published: March 27, 2021

doi:10.5539/hes.v11n2p120

URL: https://doi.org/10.5539/hes.v11n2p120

\begin{abstract}
An adequate number of healthcare providers is an essential factor in the prosperity of a population. One challenge faced by universities is student dropout. This case-control study aimed to examine the academic, psychological, medical, social, as well as female-related risk factors at a health-sciences university in Saudi Arabia in the academic year 2016-2017. The study included a total of 723 students, of whom 143 dropped out. A validated questionnaire was used to assess risk factors. Comparisons were made using chi-square test with the outcome of interest being dropout at the end of the academic year. Around $20 \%$ of students had dropped out by the end of the academic year 2016-2017. Significant risk factors for dropout included male gender, lack of previous university degree, having a primary as well as a secondary specialty choice, not matching into the first specialty choice, English language, and female-related risk factors, such as pregnancy. Health-care education is an inherently stressful environment where dropout is a concerning phenomenon. It is imperative to recognize risk factors and develop strategies to ensure students' successful adaptation and progress. Policymakers should be aware of the impact of academic and gender-related factors to address and help limit the number of students dropping out of highly needed professions.
\end{abstract}

Keywords: dropout, education, health, student, university

\section{Introduction}

The presence of an adequate number of healthcare providers is an essential factor in a population's health, to which universities can contribute by offering high-quality healthcare education to a large number of students (Anand \& Bärnighausen, 2004; Ricketts TC \& Holmes, 2007). However, the cases of students dropping out due to academic, psychological, or social factors remain a challenge for universities (O'Neill, Wallstedt, Eika, \& Hartvigsen, 2011). College dropouts have not only wasted their time in college but may have experienced psychological and social problems, which may have gone unnoticed at an early stage when an intervention may have been successful. Universities' reputation can also be affected, with consequent financial implications (O’Neill et al., 2011; Diaz \& De León AT, 2006).

Rate of medical students' dropout was estimated to range from $2 \%$ to $26 \%$, with an average dropout ratio of $11 \%$ (O'Neill et al., 2011). A study reported a 14\% dropout rate for medical students in the United Kingdom (Simpson \& Budd, 1996). A 10-year retrospective study estimated the overall attrition rate in a medical school in Ireland to be 7\%, with annual rates ranging from $4 \%$ to $9 \%$ (Maher et al., 2013). Medical students mainly drop out during their first academic year (Maher et al., 2013; Yates, 2012). Student dropout is potentially preventable and identifying its causes is essential for providing solutions. Factors that may play a role include academic performance, demographic characteristics, psychological and social problems, and physical illness or disabilities (Holt et al., 2014). Parents' educational level and financial status may also be influential (Simpson \& Budd, 1996; Maher et al., 2013). Of these factors, poor academic performance is the single most significant research finding predicting college dropout. Local studies in Saudi Arabia have mainly focused on the dropout rate or the students' 
perceptions regarding the reasons for dropout (Aldosary \& Garba, 1999; Bakhsh, 2008; Althubaiti \& Alkhazim, 2014). A study conducted at King Fahd University of Petroleum and Minerals in Dhahran concluded that students assume that poor academic performance, the courses' requisites, and the instructor of a specific course were fundamental factors in the decision making of whether to persist or drop out of a course (Aldosary \& Garba, 1999). Another study reported that between 1995 and 2004, only 62\% $(8,260)$ of medical students qualified as medical practitioners, attributing the gap to dropouts or transfers (Althubaiti \& Alkhazim, 2014). A study conducted at King Abdulaziz University estimated the medical school attrition rate from 1996 to 2002 to be $21 \%$ and found that over half (52\%) of those who dropped out did so for academic reasons (Bakhsh, 2008).

Investigating the reasons for dropping out of college is pivotal for supporting the development of prevention strategies. The aim of this study was to identify the reasons why students drop out college by investigating the psychological, social, economic, institutional, and academic factors in King Saud bin Abdulaziz University for Health Sciences (KSAU-HS) in Riyadh.

\section{Method}

This is a case-control study that was conducted at KSAU-HS that included all first-year students enrolled in the academic year of 2016-2017. The case group consisted of students who dropped out voluntarily, and the control group consisted of students who did not drop out during the same academic year. The university comprises six colleges: College of Medicine, Dentistry, Pharmacy, Applied Medical Sciences, Nursing, and Public Health and Health Informatics. University students are divided into two streams - Stream I is the undergraduate pathway that enrolls high-school graduates, in which they go through a preparatory year in order for them to choose a specialty out of the six colleges at the end of the first year. Stream II is the postgraduate pathway that enrolls bachelor's degree holders directly to their chosen specialty.

We designed a questionnaire to explore the demographic, academic, psychological, medical, social, and gender-related factors potentially contributing to dropping out. Content validity was confirmed by experts in medical education, a psychologist, and a statistician. A pilot study was conducted with 119 students (51 cases and 68 controls) to validate the questionnaire, which was edited after performing a Cronbach's alpha reliability test. The study was approved by the Institutional Review Board (IRB) of the King Abdullah International Medical Research Center. Data entry, cleaning, and analysis were performed using IBM SPSS Statistics for Windows, version 22.0 (released 2013; IBM, Armonk, NY, USA). Categorical data were presented as frequencies and percentages, and a chi-square test was used to assess the association between different categorical factors. A value of $p<0.05$ was considered statistically significant.

\section{Results}

\subsection{Baseline Characteristic}

Out of 1194 enrolled and 259 dropout students during the 2016-2017 academic year, 580 and 143 students responded to our survey, respectively. The dropout rate in the study was $20 \%$ (144). No significant relationship was found between age and dropping out. A substantial majority (68\%) of the dropouts were males. Demographic information is displayed in Table 1.

Table 1. Students' demographics

\begin{tabular}{lllll}
\hline Factor & Item & Retained N (\%) & Dropout N (\%) & P-value \\
\hline Age & 18 & $157(28)$ & $31(22)$ & $0.199^{*}$ \\
& 19 & $344(62)$ & $80(58)$ & \\
& 20 & $48(9)$ & $18(13)$ & \\
& $21+$ & $7(1)$ & $10(7)$ & \\
Gender & Male & $256(45)$ & $95(68)$ & $<0.001$ \\
& Female & $311(55)$ & $44(32)$ & \\
Stream & Stream I & $442(91)$ & $131(97)$ & 0.017 \\
& Stream II & $45(9)$ & $4(3)$ & \\
\hline
\end{tabular}

*p-value was calculated based on group comparisons: age: $<19$ vs. $\geq 19$ years of age.

\subsection{Social and Financial Factors}

Family pressure was an important factor affecting students' decision to drop out, with a total of $40 \%$ of the case group agreeing that it affected their decision to drop out. No significant association was found between income and dropout. Interestingly, students who had friends who smoked were more likely to drop out $(\mathrm{p}<0.001)$. 


\subsection{Academic Factors}

Students who indicated a first specialty choice they want to pursue were more likely to drop out. Comparing pursuing medicine as a specialty choice versus other healthcare professions was not significantly associated with dropout. The association between having a first specialty choice and dropout was statistically significant $(\mathrm{P}=.041)$. The striking difference was that students in the control group were more willing to continue despite not being matched to their first choice, with only $17 \%$ indicating that they would drop out if they did not match to their first choice. English language was a significant determinant of students' dropout, as most students indicated that their dropout decision was affected by their proficiency level in the English $(\mathrm{P}<0.001)$.

\subsection{Psychological and Physical Well-being}

Psychological and physical well-being factors played a role in the decision to drop out. Interestingly, $40 \%$ of the control group reported mental health issues compared with $20 \%$ in the case group $(\mathrm{P}<0.001)$. There was a significant relationship between bullying/harassment and dropout, with $39 \%$ of the students agreeing that such an issue influenced their decision $(\mathrm{P}<0.001)$. Health-promoting activities, such as exercise, affected the dropout outcome. Lack of exercise (zero hours per week) was reported by $29 \%$ in the dropout group and $50 \%$ in the control group $(\mathrm{P}<0.001)$. Social, academic, and psychological and physical well-being risk factors are shown in Table 2 .

Table 2. Risk factors for dropout

\begin{tabular}{lllll}
\hline Did you have a first specialty choice? & Yes & $494(95)$ & $124(90)$ & 0.041 \\
& No & $28(5)$ & $14(10)$ & \\
Did you withdraw after you did not get & Yes & $60(17)$ & $58(59)$ & $<0.001$ \\
accepted in your first specialty choice? & No & $288(83)$ & $41(41)$ & \\
What is your first specialty choice? & Medicine & $367(66)$ & $81(60)$ & $0.399 *$ \\
& Dentistry & $59(11)$ & $21(15)$ & \\
& Pharmacy & $42(8)$ & $9(7)$ & \\
& Nursing & $34(6)$ & $5(4)$ & \\
& Applied Sciences & $45(8)$ & $10(7)$ & \\
& Others & $8(1)$ & $10(7)$ & \\
Did you take any English & Yes & $209(36)$ & $64(45)$ & 0.046 \\
courses outside of college? & No & $367(64)$ & $77(55)$ & \\
Do your friends smoke? & Yes & $88(15)$ & $41(31.5)$ & $<0.001$ \\
& No & $484(85)$ & $89(68.5)$ & \\
Did you suffer from any & Yes & $211(40)$ & $28(20)$ & $<0.001$ \\
mental health issues? & No & $308(60)$ & $111(80)$ & \\
Do you have any health problems? & Yes & $69(13)$ & $20(14)$ & 0.589 \\
& No & $476(87)$ & $119(86)$ & \\
Do you think that exercise can affect & Disagree & $309(54)$ & $97(69)$ & $<0.001$ \\
academic performance positively? & Agree & $263(46)$ & $43(31)$ & \\
How many hours do & None & $286(50)$ & $41(29)$ & $<0.001 * *$ \\
you exercise per week? & Less than 2.5 hours/week & $163(28)$ & $45(32)$ & \\
& $2.5-5$ hours/ week & $78(14)$ & $40(29)$ & \\
& $>5$ hours/week & $42(7)$ & $14(10)$ & \\
\hline
\end{tabular}

*P-value was calculated; First specialty choice: medicine vs. other specialties.

** P-value was calculated exercise: $<2.5$ hours/week vs. $\geq 2.5$ hours/week.

\subsection{Female-specific Factors}

Four questions in our questionnaire explored female-specific factors: marital status, pregnancy and/or childcare, guardian refusal, and perceptions of studying as unimportant. All four elements were significantly associated with dropout $(\mathrm{p}<0.001)$.

\section{Discussion}

This study contributes to the body of knowledge about the phenomenon of dropout from a health sciences university in a Middle-Eastern context. The impact of students' withdrawal is multilayered, with financial, academic, and institutional implications. A radical solution must be sought to comprehensively address this issue. 
The factors that contribute to dropout are mainly dependent on admission criteria and the standards of the courses themselves. Students drop out from college either because they are not selected appropriately (i.e., admission selection errors) or because of difficulty adapting to and succeeding in the course itself (i.e., standards of the course).

\subsection{Demographic Characteristics}

Of the demographic variables, gender and stream (high school graduates vs. bachelor's degree holders) were the most important predictors of dropping out. Male students were at a higher risk of dropping out compared to their female counterparts. This finding is in line with a study in a military medical program that demonstrated a higher risk of dropout in male students (Stetto, Gackstetter, Cruess, \& Hooper, 2004). In our study's context, unlike female students, male students are entitled to drop out of the university without parental approval. Although the deanship of students' affairs strives to involve families in the decision process regardless of gender, male students are allowed to sign and leave university without their parents' permission, hence, there is less hindrance to male students who decide to drop out compared to female students. Age was not associated with dropout in the present study, even though it has led to conflicting results in many studies. A literature review reported that the evidence related to age as a factor of dropping out is inconsistent (O'Neill et al., 2011). One study in the UK found that students who were older than 21 years were more likely to drop out (Arulampalam, Naylor, \& Smith, 2007). However, younger age was reported in some studies to have a significant correlation with dropout (Maher et al., 2013).

Stream I students had a higher risk of dropping out than Stream II students. This finding indicates that those who studied at a university before were at a significantly lower risk. Stream II students are academically more mature and familiar with the university atmosphere. In addition, they had already been enrolled in their specialty choice, which alleviated the stress of matching into a specialty that stream I students encounter.

\subsection{Social Factors}

Overall, social factors, such as parental education and income did not appear to have a significant correlation with dropout. Our findings are consistent with a literature review that showed that the effect of such factors was modest moderate or even nonexistent in 10 of the 13 studies included (O'Neill et al., 2011). Another retrospective study also reported that socio-demographic factors were not significantly associated with dropping out (Maher et al., 2013). Family pressure was an identified risk factor for dropout, as students who dropped out mentioned that their decision was influenced by family pressure. This indicates the importance of social factors in the population.

\subsection{Academic Factors}

Unsurprisingly, academic factors were a major contributor to students' dropout in our study. This has been well demonstrated in the literature in several studies. In a 10-year retrospective attrition rate study, $56 \%$ of the students who dropped out faced academic challenges (Maher et al., 2013). The nature of health science colleges is competitive and stressful. Therefore, intervening with career counseling and academic support for targeted students is invaluable in limiting the number of dropouts. The academic challenges are compounded by the language barrier for non-native speakers, as the teaching medium is English, even for core scientific subjects. Such subjects are challenging, as indicated by a study that found that students at scientific colleges had a higher dropout rate than humanities colleges (Al Ghanboosi, 2013). The same study also highlighted challenges related to language and the multicultural background of the instructors at Sultan Qaboos University and Kuwait University. Education in both of these Gulf countries is similar in context to the university we conducted the study at. Therefore, students with low English language admission test scores can also be targeted to improve their language skills from the beginning of the year.

\subsection{Psychological and Physical Well-being}

It is believed that the psychological reasons are underreported and not thoroughly studied, as indicated in the literature (O'Neill et al., 2011; Maher et al., 2013). Mental health issues were less reported by students who dropped out. Several reasons can explain this finding. Students who fear being stigmatized may be less willing to admit or seek professional help, which could be seen as a sign of failure (Martin, 2010; Wada et al., 2019). However, such mental issues persist and eventually impact student performance and initiate a vicious cycle. Despite the well-known positive effects of physical activity on psychological and physical well-being, there is a clear association between dropping out and increased physical activity. In fact, people who continued their studies had significantly more sedentary lifestyles and less exercise hours per week compared to those who dropped out. Previous studies have showed mixed results with regard to physical activity and academic 
performance (Al-Drees et al., 2016; Calestine, Bopp, Bopp \& Papalia, 2017). In one study, college students had more sedentary lifestyles due to their packed schedules. Achieving a higher GPA was linked to an increased BMI (Calestine et al., 2017). There has also been a clear correlation between a decrease in frequency of exercise after enrollment in college in one Saudi study (Alkhateeb et al., 2019).

\subsection{Female-specific Factors}

Female students faced specific challenges such as marriage, pregnancy and childcare, guardian refusal, and perceptions of tertiary education as unnecessary, which were significantly associated with dropout. We propose that some of these factors are unique to the Middle-Eastern context and similar cultures. The fact that a female student cannot be admitted to or drop out of college without her guardian's approval is essential for interpreting the data. One may argue that such a factor would add a barrier to leaving college, explaining the lower dropout rate for female students. However, our findings suggest that it played a significant role in dropping out, as the majority agreed that guardian disapproval of their studies affected their decision to drop out. This challenge 5indicates the importance of empowering female students to make the decision about their education without the need for guardians' approval. Most female students reported that perceiving tertiary education as a trivial matter was a factor in their decision to leave college. This highlights a significant cultural background, indicating the need for an intervention to ensure female students' educational future. However, female students could have realized that they are victims of a socially embedded conflict between their role as students and their role as housewives, which pressures them to drop out of college and assume their socially acceptable roles. This phenomenon was investigated in an Iranian study, where female students reported that they suffered from a lack of family support to continue their studies (Moghadam, Khiaban, Esmaeili \& Salsali, 2017). Marriage, pregnancy, and childcare were also considered important in their decision to drop out. Studentship and motherhood do not necessarily have conflicting roles. Yet, in the current social structure, the perceived incompatibility between these roles can be a burden, causing feelings of guilt and a negative self-image if students feel that they are neglecting their children in order to attend classes. Such students can be supported through college administration, teaching staff, and college resources (Moghadam et al., 2017).

\subsection{Limitations}

The findings of this study are comparable to findings regarding other institutions. However, our results are subject to certain limitations. We included only one institution for one academic year. The controls were not followed up, and thus we did not examine the students who dropped out after that academic year. However, we chose first-year students as the control group since the majority of students who leave college are typically first-year students, as reported by Maher et al. (2013) and Al Ghanboosi (2013).

\subsection{Recommendations}

A national study to further understand and address the subtle risk factors for dropout is essential. Although different universities face different challenges with their students, the main factors presented in this study and other studies are critical to address. Given that academic factors pose a substantial risk for dropout, mentorship programs need to be more proactive in addressing academic obstacles. As students struggle to study courses in a second language, summer bootcamps prior to starting university for targeted students who have lower English language test scores can be beneficial. Furthermore, establishing student-wellness centers to aid students in coping strategies and addressing mental health issues is recommended. To help reduce the amount of stress and rate of dropout among female students, leaders may consider establishing new policies to help support female students and adjustment of their courses per individual needs (e.g., pregnancy).

\section{Conclusion}

Nearly one-fifth of the population we studied dropped out, creating a major concern for decision makers to identify risk factors for dropout. Our study suggests that there are multiple factors that lead students to drop out, including male gender, female-specific factors, performance in English language, lack of an undergraduate degree, and mental health issues. Involving policy makers to implement preventive measures, such as mentorship programs, to reduce the number of students dropping out and make informed decisions to adjust the risk factors is highly needed.

\section{Disclosure}

Ethical approval has been granted on $1^{\text {st }}$ of May 2017 by King Abdullah International Medical Research Center (KAIMRC) with a reference number: IRBC/528/17. 


\section{Acknowledgments}

We would like to acknowledge the efforts of Prof. Raghib Abu-Saris and Dr. Emad Masuadi for their critique of the statistical part of the methodology and the development of the questionnaire. We would also like to acknowledge the efforts of Prof. Mohi Eldin Magzoub for his critique of our development of the paper's conceptual framework.

\section{References}

Aldosary, A. S., \& Garba, S. B. (1999). An Analysis of Factors Contributing to College Student Dropout in a Medium Sized Technical University: The Case of The King Fahd University of Petroleum and Minerals Dhahran, Saudi Arabia. Higher Education Policy, 12(4), 313-328. https://doi.org/10.1016/S0952-8733(99)00021-5

Al-Drees, A., Abdulghani, H., Irshad, M., Baqays, A. A., Al-Zhrani, A. A., Alshammari, S. A., \& Alturki, N. I. (2016). Physical Activity and Academic Achievement among The Medical Students: A Cross-Sectional Study. Medical Teacher, 38(sup1), S66-S72. https://doi.org/10.3109/0142159X.2016.1142516

Al Ghanboosi, S. (2013). Factors Influencing Students' Attrition at Sultan Qaboos University (SQU). Education, 133(4), 513-524.

Alkhateeb, S. A., Alkhameesi, N. F., Lamfon, G. N., Khawandanh, S. Z., Kurdi, L. K., Faran, M. Y., ... Safdar, O. Y. (2019). Pattern of Physical Exercise Practice among University Students in The Kingdom of Saudi Arabia (before beginning and during college): A Cross-Sectional Study. BMC Public Health, 19(1), 1716. https://doi.org/10.1186/s12889-019-8093-2

Anand, S., \& Bärnighausen, T. (2004). Human Resources and Health Outcomes: Cross-Country Econometric Study. The Lancet, 364(9445), 1603-1619. https://doi.org/10.1016/S0140-6736(04)17313-3

Arulampalam, W., Naylor, R. A., \& Smith, J. P. (2007). Dropping Out of Medical School in the UK: Explaining the Changes Over Ten years. Medical Education, 41(4), 385-394. https://doi.org/10.1111/j.1365-2929.2007.02710.x

Althubaiti, A., \& Alkhazim, M. (2014). Medical Colleges in Saudi Arabia: Can We Predict Graduate Numbers? Higher Education Studies, 4(3), 1-8. http://dx.doi.org/10.5539/hes.v4n3p1

Bakhsh, T. M. (2008). Analysis of Attrition Over a 7-year Period at The Faculty of Medicine, King Abdulaziz University, Jeddah. Journal of King Abdulaziz University - Medical Sciences, 15(4), 49-57. https://doi.org/10.4197/med.15-4.5

Calestine, J., Bopp, M., Bopp, C. M., \& Papalia, Z. (2017). College Student Work Habits are Related to Physical Activity and Fitness. International Journal of Exercise Science, 10(7), 1009-1017.

Diaz, P., \& De León, A. T. (2016). Design and Validation of a Questionnaire to Analyze University Dropout-ADES. World Journal of Education Research, 3(2), 267-280. https://doi.org/10.22158/wjer.v3n2p267

Holt, M. K., Green, J. G., Reid, G., DiMeo, A., Espelage, D. A., Felix, E. D., ... Sharkey, J. D. (2014). Associations Between Past Bullying Experiences and Psychosocial and Academic Functioning Among College Students. Journal of American College Health, 62(8), 552-560. https://doi.org/10.1080/07448481.2014.947990

Maher, B. M., Hynes, H., Sweeney, C., Khashan, A. S., O’Rourke, M., Doran, K., ... O’Flynn, S. (2013). Medical School Attrition-beyond the Statistics a ten-year Retrospective Study. BMC Medical Education, 13(13). https://doi.org/10.1186/1472-6920-13-13

Martin, J. M. (2017). Stigma and student mental health in higher education. Education Research and Development, 29(3), 259-74. https://doi.org/10.1080/07294360903470969

Moghadam Z. B., Khiaban M. O., Esmaeili M., \& Salsali M. (2017). Motherhood Challenges and Well-being along with the Studentship Role among Iranian Women: A Qualitative Study. International Journal of Qualitative Studies on Health and Well-being, 12(1). https://doi.org/10.1080/17482631.2017.1335168

O’Neill, L., Wallstedt, B., Eika, B., \& Hartvigsen, J. (2011). Factors Associated with Dropout in Medical Education: A Literature Review. Medical Education, 45(5), 440-454. https://doi.org/10.1111/j.1365-2923.2010.03898.x

Ricketts, T. C., \& Holmes, G. M. (2007). Mortality and Physician Supply: Does Region Hold the Key to the 
Paradox? Health Services Research, 42(6p1), 2233-2251. https://doi.org/10.1111/j.1475-6773.2007.00728.x

Simpson, K. H., \& Budd, K. (1996). Medical Student Attrition: A 10-year Survey in One Medical School. Medical Education, 30(3), 172-178. https://doi.org/10.1111/j.1365-2923.1996.tb00739.x

Stetto, J. E., Gackstetter, G. D., Cruess, D. F., \& Hooper, T. I. (2004). Variables Associated with Attrition from Uniformed Services University of the Health Sciences Medical School. Military Medicine, 169(2), 102-107. https://doi.org/10.7205/MILMED.169.2.102

Wada, M., Suto, M. J., Lee, M., Sanders, D., Sun, C., Le, T. N., ... Chauhan S. (2019). University students' Perspectives' on Mental Illness Stigma. Mental Health Preview, 14, 200159. https://doi.org/10.1016/j.mph.2019.200159

Yates, J. (2012). When Did They Leave, and Why? A Retrospective Case Study of Attrition on The Nottingham Undergraduate Medical Course. BMC Medical Education, 12(43). https://doi.org/10.1186/1472-6920-12-43

\section{Copyrights}

Copyright for this article is retained by the author(s), with first publication rights granted to the journal.

This is an open-access article distributed under the terms and conditions of the Creative Commons Attribution license (http://creativecommons.org/licenses/by/4.0/). 